\title{
BAETIDAE (INSECTA: EPHEMEROPTERA) DE NOVA XAVANTINA, MATO GROSSO, BRASIL: NOVOS REGISTROS E DESCRIÇÃO DE UMA NOVA ESPÉCIE DE CLOEODES TRAVER
}

\author{
Frederico Falcão Salles ${ }^{1,2}$; Joana Darc Batista ${ }^{3}$ \& Helena Ramos Soares Cabette ${ }^{3}$
}

Biota Neotropica v4 (n2) - http://www.biotaneotropica.org.br/v4n2/pt/abstract?article+BN02404022004

\author{
recebido em: $19 / 4 / 2004$ \\ revisado em: $18 / 6 / 2004$ \\ publicado em: 01/07/2004
}

1. Universidade Federal de Viçosa. Museu de Entomologia, Departamento de Biologia Animal, Viçosa, Minas Gerais, Brasil.

www.insecta.ufv.br/Entomologia/cien/sistematica/ephemeroptera/ephembrasil/index.htm

2. Autor correspondente. E-mail: ffsalles@insecta.ufv.br

3. Universidade do Estado de Mato Grosso, Departamento de Ciências Biológicas, 78690-000, Nova Xavantina, MT, Brasil.

\begin{abstract}
Studies carried mainly in the municipality of Nova Xavantina, Mato Grosso State, have lead us to the discovery of several species of Baetidae. While one of them represents a new species of the genus Cloeodes and is herein described, the others represent new records for the state or even for Brazil. The new species, Cloeodes auwe sp. nov., can be differentiated from the other known species of the genus by the following characteristics: body color pattern, long and two-segmented maxillary palp, third segment of labial palp robust and apically truncate, long tarsal claws (0.5 times the length of the respective tarsus), hind wing pads absent, and number of spines on the posterior margin of the third tergite and on the paraproct. Besides this new species, the following taxa of Baetidae were also found: Adebrotus amazonicus, Americabaetis alphus, Apobaetis sp., Aturbina georgei, Baetodes sp., Callibaetis sp.1, Callibaetis sp.2, Camelobaetidius janae, Cryptonympha sp., Harpagobaetis gulosus, Paracloeodes binodulus, Spiritiops silvudus, Waltzoyphius fasciatus, and Zelusia principalis.
\end{abstract}

Key words: Ephemeroptera, Baetidae, Cloeodes auwe sp. nov., Brazil, West-Central, new records.

\section{Resumo}

Estudos realizados principalmente no município de Nova Xavantina, Mato Grosso, levaram-nos à descoberta de diversas espécies de Baetidae. Enquanto uma delas representa uma nova espécie de Cloeodes, aqui descrita, as outras representam novos registros para o estado ou até mesmo para o Brasil. A nova espécie, Cloeodes auwe sp. nov., pode ser diferenciada das demais espécies do gênero pela seguinte combinação de caracteres: padrão de coloração corporal; palpo maxilar longo, porém claramente com dois artículos; terceiro artículo do palpo labial robusto e de ápice truncado; garras relativamente longas ( 0,5 vezes o comprimento do respectivo tarso), tecas alares posteriores ausentes e número de espinhos na margem posterior do tergito 3 e paraprocto. Além dessa espécie, os seguintes táxons de Baetidae também foram encontrados: Adebrotus amazonicus, Americabaetis alphus, Apobaetis sp., Aturbina georgei, Baetodes sp., Callibaetis sp.1, Callibaetis sp.2, Camelobaetidius janae, Cryptonympha sp., Harpagobaetis gulosus, Paracloeodes binodulus, Spiritiops silvudus, Waltzoyphius fasciatus e Zelusia principalis.

Palavras-chave: Ephemeroptera, Baetidae, Cloeodes auwe sp. nov., Brasil, Centro-Oeste, novos registros.

http://www.biotaneotropica.org.br 


\section{Introdução}

O conhecimento a respeito da fauna de Baetidae (Insecta: Ephemeroptera) do Estado do Mato Grosso, assim como os demais estados da Região Centro-Oeste, restringese a um pequeno número de táxons. As únicas espécies da família formalmente registradas para o Mato Grosso são: Apobaetis fiuzai Salles \& Lugo-Ortiz, 2002, Aturbina georgei Lugo-Ortiz \& McCafferty, 1996, Cloeodes hydation McCafferty \& Lugo-Ortiz, 1995 e Varipes helenae Salles \& Batista, 2004 (Lugo-Ortiz \& McCafferty 1996a, McCafferty \& Lugo-Ortiz 1995, Nolte et al. 1997, Salles \& Batista 2004, Salles \& Lugo-Ortiz 2002a). Além dessas espécies, Nolte et al. (1997), ao abordarem aspectos ecológicos sobre os Ephemeroptera do Rio Bento Gomes, também reportaram sobre algumas espécies, a maioria ainda não determinada, para o estado.

Apesar disso, estudos em andamento realizados em diversos ambientes aquáticos do município de Nova Xavantina e arredores, sudeste do Mato Grosso, vêm demonstrando que a fauna de Baetidae da região é bem diversificada. Varipes Lugo-Ortiz \& McCafferty, 1998, por exemplo, foi pela primeira vez reportado para o Brasil, demonstrando que para um conhecimento mais aprofundado acerca da fauna brasileira de Baetidae, maiores esforços se fazem necessários para esse estado (Salles \& Batista, 2004). No presente trabalho, dando continuidade ao levantamento da fauna de Baetidae da região e, conseqüentemente do Brasil, são apresentados registros de treze táxons da família, a maioria inéditos para o estado. Além disso, também é apresentada a descrição de uma nova espécie do gênero Cloeodes Traver, 1938.

\section{Material e Métodos}

As coletas, de caráter qualitativo, foram realizadas em diversos rios e córregos pertencentes à Bacia do Rio das Mortes e localizados nos municípios de Barra do Garças e, principalmente em Nova Xavantina, ambos a leste do Mato Grosso (Fig. 1). Os registros, assim como a descrição da nova espécie de Cloeodes, são apresentados em ordem alfabética. O material encontra-se depositado na coleção de Ephemeroptera, Laboratório de Entomologia, Departamento de Zoologia, Instituto de Biologia, Universidade Federal do Rio Janeiro, Rio de Janeiro, Brasil.

\section{Resultados}

1995

Adebrotus amazonicus Lugo-Ortiz \& McCafferty,

MATERIAL EXAMINADO: município de Nova Xavantina, Rio Pindaíba, Pontal, 02-X-2003, 14²7'0"'S / $51^{\circ} 42^{\prime} 26^{\prime \prime} \mathrm{W}, 1$ ninfa, J.D. Batista.

COMENTÁRIOS: Desde a época de sua descrição, o gênero monotípico Adebrotus Lugo-Ortiz \& McCafferty, 1995 só havia sido registrado, além da localidade-tipo de sua única espécie conhecida (Amazonas, Brasil), para a Guiana Francesa e baseado em um indivíduo não nomeado (LugoOrtiz \& McCafferty 1995, Orth et al. 2000). A espécie e o gênero são, conseqüentemente, reportados pela primeira vez para a Região Centro-Oeste do Brasil, estendendo ao sul sua distribuição prévia.

Americabaetis alphus Lugo-Ortiz \& McCafferty, 1996

MATERIAL EXAMINADO: município de Nova Xavantina, Rio Pindaíba, Pontal, 02-X-2003, 14²7'0'S / 5142'26'W, 2 ninfas, J.D. Batista; município de Nova Xavantina, Ponte de Pedra, 03-ix-2003, 14º1'08'S / 52 41'13" W, 1 ninfa, F.F. Salles, J.D. Batista, H.R.S. Cabette.

COMENTÁRIOS: Espécie de ampla distribuição na América do Sul, A. alphus se encontra registrada no Brasil para quase todos os estados das regiões Sul e Sudeste (Lugo-Ortiz \& McCafferty 1996c, Francischetti et al. 2003, Salles et al. 2003b). A espécie é, portanto, pela primeira vez reportada para a Região Centro-Oeste, estendendo ao norte sua distribuição. O gênero também é pela primeira vez reportado para a região, apesar de que Acerpenna sp., registrada por Nolte et al. (1997) para o Rio Bento Gomes, provavelmente pertença a Americabaetis (para maiores detalhes veja Lugo-Ortiz \& McCafferty 1996c).

\section{Apobaetis sp.}

MATERIAL EXAMINADO: município de Nova Xavantina, Rio Pindaíba, Pontal, 23-ix-2003, 14²7'0"S / 51'42’26,”W, 1 ninfa, J.D. Batista, H.R.S. Cabette.

COMENTÁRIOS: Previamente reportado para o Estado do Mato Grosso, Apobaetis Day, 1955 está representado na América do Sul por duas espécies, A. fiuzai e A. signifer Lugo-Ortiz \& McCafferty, 1997 (Lugo-Ortiz \& McCafferty 1997, Salles \& Lugo-Ortiz 2002a). Já a espécie encontrada, não se refere a nenhuma das duas citadas. No entanto, como apenas uma ninfa foi encontrada e em mau estado de conservação, sua descrição não é apresentada.

\section{Aturbina georgei Lugo-Ortiz \& McCafferty, 1996}

MATERIAL EXAMINADO: município de Nova Xavantina, Córrego Antártico, 06-ix-2003, 1450'51' S / 52 $37^{\circ} 20^{\prime \prime W}, 1$ ninfa, F.F. Salles, J.D. Batista, H.R.S. Cabette.

COMENTÁRIOS: Outra espécie de ampla distribuição (veja Salles et al. 2003b), A. georgei já se encontrava registrada para o Mato Grosso, porém apenas no centro-sul do estado (Lugo-Ortiz \& McCafferty 1996a, Nolte et al. 1997). A presença de $A$. georgei em Nova Xavantina estende à leste sua distribuição no estado. 


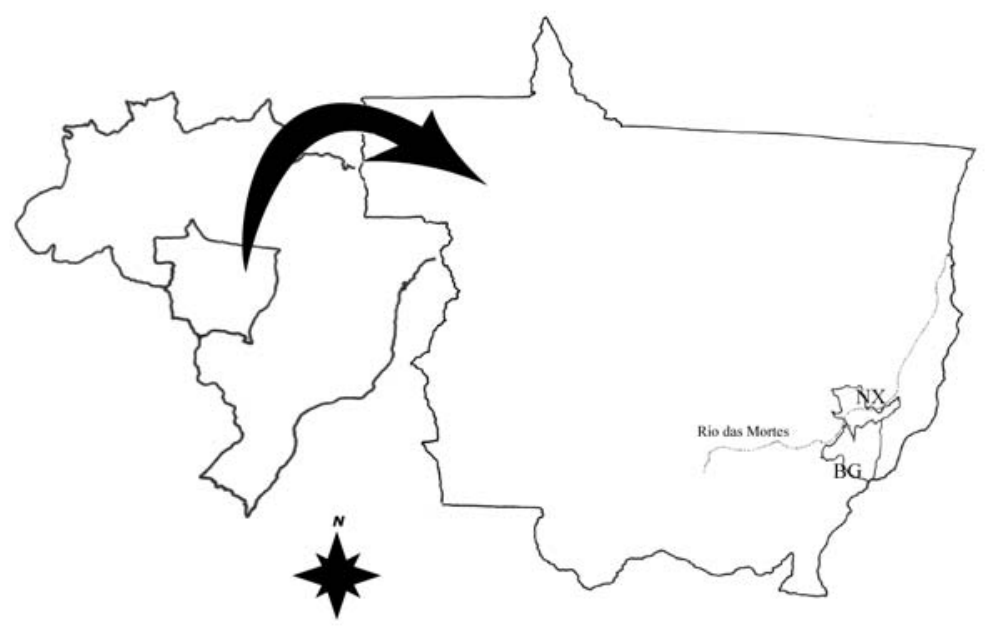

Figura 1. Mapas do Brasil e do Estado do Mato Grosso mostrando a localização dos municípios de Nova Xavantina (NX) e Barra do Garças (BG), além do Rio das Mortes (pontilhado).

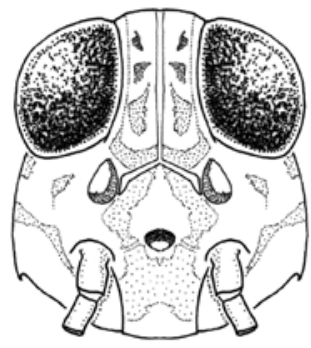

2

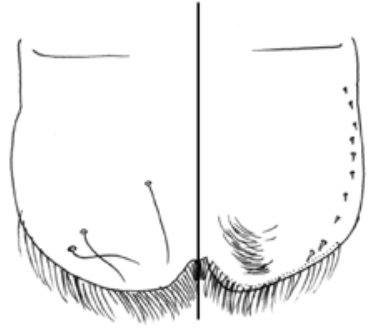

3

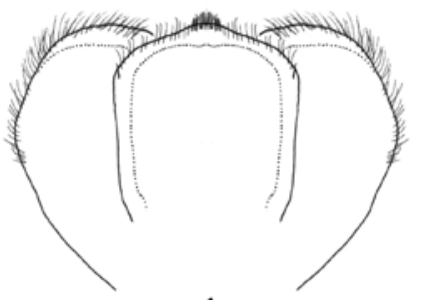

4
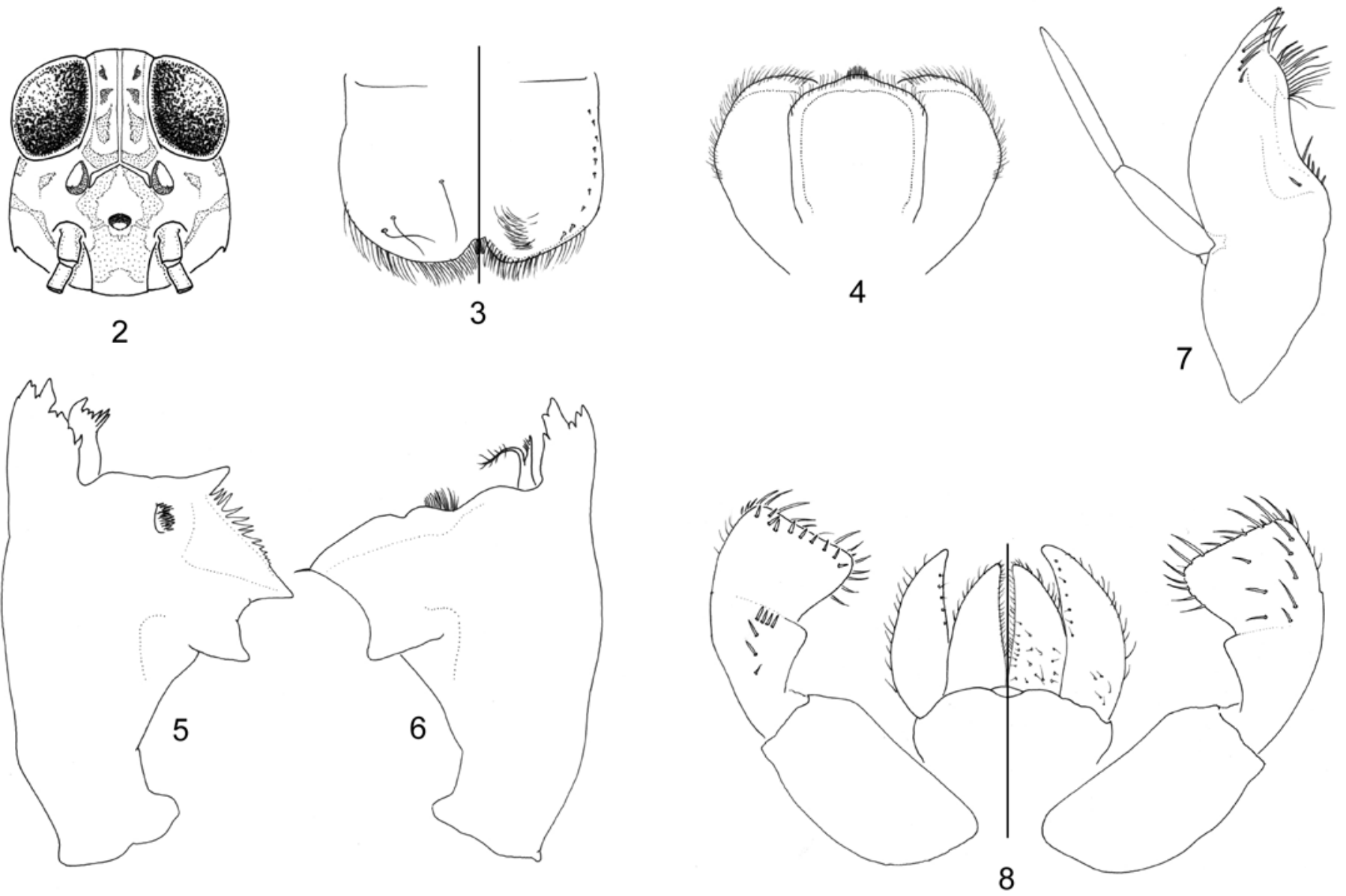

Figuras 2-8. Cloeodes auwe sp. nov. ninfa. 2. Cabeça (frontal). 3. Labro (esquerda, dorsal - direita, ventral). 4. Hipofaringe. 5. Mandibula esquerda. 6. Mandibula direita. 7. Maxila. 8. Lábio (esquerda, dorsal - direita, ventral).

http://www.biotaneotropica.org.br 

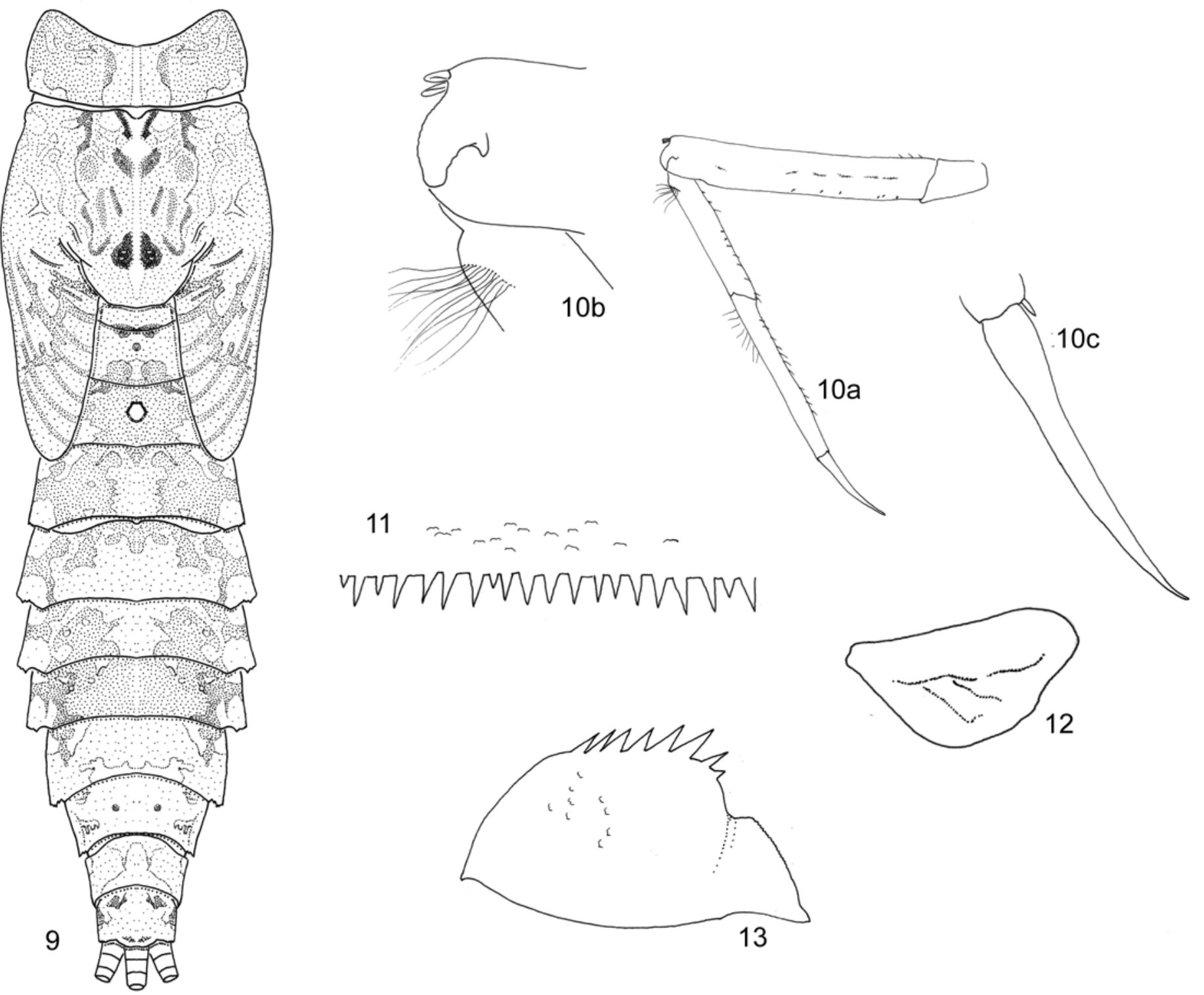

Figuras 9-13. Cloeodes auwe sp. nov. ninfa. 9. Tórax e abdome (dorsal). 10a. Perna anterior. 10b. Detalhe do ápice do fêmur e ápice da tíbia anterior. 10c. Detalhe da garra anterior. 11. Tergito 3 (margem posterior). 12. Brânquia 4. 13. Paraprocto. 


\section{Baetodes sp.}

MATERIAL EXAMINADO: município de Nova Xavantina, Córrego Antártico, pedra em correnteza, 06-ix2003, 14 50 50'51”'S / 52³7’20 “W, 10 ninfas, F.F. Salles, J.D. Batista.

COMENTÁRIOS: Registrado pela primeira vez para toda a Região Centro-Oeste, o gênero Baetodes Needham \& Murphy, 1924 encontra-se representado no Brasil por três espécies descritas, atualmente restritas às regiões Sul e Sudeste (Needham \& Murphy 1924, Demoulin 1955, Traver 1944, Mayo 1972). Como as descrições de algumas dessas espécies são incompletas ou antigas, um estudo mais detalhado precisa ser realizado para a identificação correta de seus integrantes.

\section{Callibaetis sp. 1 e Callibaetis sp. 2}

MATERIAL EXAMINADO: $C$. sp.1, município de Barra do Garças, Taquaral, Vereda, 04-vi-2003, 1544'20,4”S / 52 23'31,6”'W, 1 ninfa, J.D. Batista, H.R.S. Cabette.; C. sp. 2, município de Nova Xavantina, Córrego Antártico, 06-ix2003, 14 50'51"S / 52³7’20 “W, 1 ninfa, F.F. Salles, J.D. Batista, H.R.S. Cabette.

COMENTÁRIOS: Previamente reportado para o estado como Callibaetis sp. (Nolte et al. 1997), o gênero Callibaetis Eaton, 1881 tem sua taxonomia baseada principalmente no estágio alado, com raras espécies descritas de ambos os estágios (e.g. C. guttatus Navás e $C$. radiatus Navás; Da-Silva 1991, Salles et al. 2003a). Apenas a partir da criação das ninfas até o estágio adulto será possível a identificação específica de seus representantes.

Camelobaetidius janae Dominique \& Thomas in Dominique, Thomas, Orth \& Dauta, 2000

MATERIAL EXAMINADO: município de Nova Xavantina, Rio Pindaíba, Pontal, 02-X-2003, 14²7'0'S / $51^{\circ} 42$ '26”W, 4 ninfas, J.D. Batista.

COMENTÁRIOS: Enquanto o gênero já se encontrava reportado para o estado como Camelobaetidius sp. (Nolte et al. 1997), C. janae é pela primeira vez registrada para o Brasil. Sua distribuição prévia estava restrita à Guiana Francesa (Dominique et al. 2000), e o presente registro a estendeu consideravelmente ao sul.

Cloeodes auwe Salles \& Batista, sp. nov. (Figs. 2-13)

NINFA. Comprimento do corpo: 5,0 - 5,4 mm. Comprimento dos filamentos caudais: $2,0 \mathrm{~mm}$. Coloração geral (Fig. 9), variando de creme a castanho com eventuais marcações castanho-escuras. Cabeça (Fig. 2): coloração geral creme com marcações variando de castanho a castanhoclaro, mais evidentes na fronte. Ocelos laterais circundados de castanho-claro, mediano circundado de negro. Antena cerca de duas vezes o comprimento da cápsula cefálica.
Labro subquadrangular (Fig. 3), 1,5 vezes mais largo que longo; dorsalmente com par submediano de cerdas longas, finas e simples e dois pares de cerdas semelhantes, situadas anterolateralmente; ventralmente com fileira de dez cerdas pequenas, robustas, simples e de ápice afilado. Hipofaringe como na Fig. 4. Mandíbula esquerda (Fig. 5) com incisivos fortemente fusionados; incisivos externos e internos com quatro e cinco dentículos respectivamente; prosteca robusta, apicalmente denteada; margem anterior desprovida de cerdas. Mandíbula direita (Fig. 6) com incisivos fortemente fusionados; incisivos externos e internos com três dentículos cada; prosteca bifurcada; ramo inferior longo e plumoso; ramo superior apicalmente denteado; margem anterior desprovida de cerdas. Maxila com protuberância mediana (Fig. 7), provida de fileira de cinco cerdas finas e simples, mais longas em direção ao ápice e uma cerda curta, robusta e apicalmente afilada; gálea-lacínia com fileira subapical de quatro cerdas longas, robustas e de ápice afilado; palpo maxilar excedendo ápice da gálea-lacínia, com dois artículos; segundo artículo 1,4 vezes o comprimento do primeiro. Lábio (Fig. 8) com densa fileira de cerdas curtas, robustas e simples ao longo das margens interna e externa da glossa; superfície dorsal da paraglossa recoberta por cerdas curtas, finas e simples na metade basal; paraglossa com fileira de cerdas curtas, robustas e simples ao longo da margem externa e na metade apical da margem interna; ventralmente com fileira submarginal de cerdas curtas, finas e simples e com pequeno grupo de cerdas semelhantes próximas à sua base; segundo artículo do palpo labial com duas fileiras de cerdas na superfície dorsal, uma transversal contendo quatro cerdas longas, robustas e simples e outra longitudinal, contendo três cerdas semelhantes às anteriores porém mais longas; terceiro artículo robusto, de ápice truncado com diversas cerdas longas, robustas e apicalmente afiladas na superfície ventral, formando duas fileiras longitudinais, na superfície dorsal formando uma fileira submarginal e na margem anterior. Tórax: coloração geral como na Fig. 9, com marcações variando de castanho a castanho-escuro. Teca alar posterior ausente. Pernas estreitas (Figs. 10a-c), de coloração esbranquiçada; fêmur com pequenas faixas escuras e estreitas com reduzido número de cerdas curtas e simples tanto na margem dorsal quanto ventral; margem dorsal dos fêmures desprovida de projeção (Fig. 10a) e com duas cerdas sublanceoladas; tíbias com arco basal de cerdas longas e finas; tarsos com fileira dorsal de cerdas longas e finas; garra (Fig. 10c) 0,5 vezes o comprimento do tarso. Abdome: coloração geral creme com marcações variando de castanhoclaro a escuro, padrão de coloração como na Fig. 9. Margem posterior do tergito 3 (Fig. 11) com 28 espinhos cerca de duas a três vezes mais longos que a largura da base. Brânquias (Fig. 12) ovaladas, margens desprovidas de cristas e com ramos das traquéias interrompidos. Paraprocto (Fig. 13) com oito espinhos. Filamentos caudais esbranquiçados, exceto por dois artículos próximos ao ápice de coloração castanha; cercos tendo, a cada quatro 
artículos, espinhos na margem externa.

ADULTO: desconhecido

MATERIAL EXAMINADO: Holótipo: ninfa fêmea, Brasil, Estado do Mato Grosso, município de Nova Xavantina, Córrego Antártico, 06-ix-2003, barranco em remanso, 1450'51”S / 52³7’20 “W, F.F. Salles, J.D. Batista. Parátipos: município de Nova Xavantina, Rio Pindaíba, Pontal, 02-x-2003, 14²7'0"'S / 51 '42'26,"W, 7 ninfas, J.D. Batista; mesma referência, exceto 23-ix-2003, 3 ninfas.

ETIMOLOGIA: o epíteto da espécie é a forma como o povo Xavante, habitantes da região onde a espécie foi coletada, se autodenomina. Deriva de A'uwê Uptabi, do tronco lingüístico Jê e significa povo verdadeiro.

COMENTÁRIOS: Cloeodes auwe sp. nov. pode ser diagnosticada das demais espécies do gênero conhecidas no estágio ninfal pela seguinte combinação de caracteres: padrão de coloração corporal (Figs. 2, 9), palpo maxilar longo, porém claramente com dois artículos (Fig. 7), terceiro artículo do palpo labial robusto e de ápice truncado (Fig. 8), garras (Figs. 10a, 10c) relativamente longas $(0,5$ vezes o comprimento do respectivo tarso), tecas alares posteriores ausentes e pelo número de espinhos na margem posterior do tergito 3 (Fig. 11) e paraprocto (Fig. 13).

Assim como outras espécies de Cloeodes de hábito conhecido [e.g. C. hydation, C. irvingi Waltz \& McCafferty e C. jaragua Salles \& Lugo-Ortiz, 2003 (McCafferty \& LugoOrtiz 1995, Lugo-Ortiz et al. 2002, Salles \& Lugo-Ortiz 2003)], ninfas de $C$. auwe foram encontradas em áreas de remanso e preferencialmente em barranco com raízes e vegetação marginal. O comprimento atípico de suas pernas e, principalmente das garras, provavelmente está associado a esse hábito.

\section{Cryptonympha sp.}

MATERIAL EXAMINADO: município de Barra do Garças, Rio Pindaíba, 26-ii-2001, Fazenda Duas Âncoras, $14^{\circ} 51^{\prime} 48,6^{\prime}$ 'S / 52 $01^{\prime}$ '02,4 “W, 3 ninfas, J.D. Batista, H.R.S. Cabette; município de Nova Xavantina, Rio das Mortes, entorno da Ilha Bela, 31-viii-2003, 1442'05”S / 52²6’39 “W, 1 ninfa, F.F. Salles, J.D. Batista, H.R.S. Cabette.

COMENTÁRIOS: Gênero reportado para diversos estados brasileiros (veja Lugo-Ortiz \& McCafferty 1998, Salles et al. 2003b, Salles \& Francischetti 2004), Cryptonympha Lugo-Ortiz \& McCafferty, 1998 está representado por apenas duas espécies descritas. A presente espécie, assim como Apobaetis sp., trata-se, também, de uma provável espécie nova, mas a quantidade de material assim como sua qualidade comprometem qualquer descrição. Apesar de amplamente distribuído, o gênero é pela primeira vez registrado para a Região CentroOeste.

\section{Harpagobaetis gulosus Mol, 1986}

MATERIAL EXAMINADO: município de Nova Xavantina, Rio Pindaíba, Pontal, 02-X-2003, 14²7'0”S / $51^{\circ} 42^{\prime} 26^{\prime \prime} \mathrm{W}, 4$ ninfas, J.D. Batista.

COMENTÁRIOS: H. gulosus, única espécie do gênero e descrita do Suriname (Mol 1986), foi recentemente registrada para o Brasil, Estado de Goiás (Salles \& LugoOrtiz 2002b). A presença da espécie em Mato Grosso era, portanto, esperada e estende ligeiramente a noroeste sua distribuição.

Paracloeodes binodulus Lugo-Ortiz \& McCafferty, 1996

MATERIAL EXAMINADO: município de Barra do Garças, Rio Pindaíba, vegetação submersa, 23-ix-2003, $14^{\circ} 53^{\prime} 30,8^{\prime}$ 'S / 52 00 '21,3”W, 1 ninfa, J.D. Batista, H.R.S. Cabette.

COMENTÁRIOS: Descrita com base em material procedente exclusivamente do Pará (Lugo-Ortiz \& McCafferty 1996b), P. binodulus é, portanto, registrada pela primeira vez para o Mato Grosso. Uma espécie não determinada de Paracloeodes já havia sido reportada para o estado, contudo restrita ao centro-sul (Nolte et al. 1997).

Spiritiops silvudus Lugo-Ortiz \& McCafferty, 1998

MATERIAL EXAMINADO: município de Nova Xavantina, Córrego do Botina, 02-xi-2003, pedra em correnteza, $14^{\circ} 44^{\prime} 23^{\prime}$ 'S / 52 33' 1'”W, 5 ninfas, F.F. Salles, J.D. Batista.

COMENTÁRIOS: S. silvudus estava previamente registrada para o Brasil, estados do Amazonas e Pará, e para a Guiana Francesa (Lugo-Ortiz \& McCafferty 1998). O presente registro estende ao sul a distribuição da espécie. Suas ninfas foram encontradas geralmente em áreas de forte correnteza, juntamente com aquelas do gênero Baetodes. Em áreas onde musgos ou representantes de Podostemaceae estavam presentes, suas ninfas foram coletadas até mesmo em superfícies verticais com forte volume d'água.

Nos indivíduos estudados, foi encontrado indicativo de um terceiro artículo no palpo maxilar, característica não abordada na descrição original. Como nenhuma outra diferença foi encontrada, supõe-se que tal caráter seja uma variação de $S$. silvudus, ou que esse indicativo tenha sido negligenciado por Lugo-Ortiz \& McCafferty (1998). Cabe ressaltar (Salles, dados não publicados) que ninfas provavelmente pertencentes à $S$. silvudus, coletadas no Estado do Amazonas, assim como ninfas de uma nova espécie do gênero, coletadas no Estado de Goiás, também apresentam o referido indicativo no palpo maxilar. 


\section{5}

$$
\text { Waltzoyphius fasciatus McCafferty \& Lugo-Ortiz, }
$$

MATERIAL EXAMINADO: município de Nova Xavantina, Ponte de Pedra, margem, 03-ix-2003, 14 01'08'S / 52 $2^{\circ} 1^{\prime} 13$ “'W, 1 ninfa, F.F. Salles, J.D. Batista, H.R.S. Cabette.

COMENTÁRIOS: Com distribuições pontuais no norte e sudeste do país (Lugo-Ortiz \& McCafferty 1995, Lugo-Ortiz et al. 2002, Salles et al. 2003b), a presença de $W$. fasciatus, apesar de constituir a primeira para a Região Centro-Oeste, já era esperada.

\section{Zelusia principalis Lugo-Ortiz \& McCafferty, 1998}

MATERIAL EXAMINADO: município de Nova Xavantina, Córrego Roda D'água, 31-viii-2003, 1443'07”S / $52^{\circ} 30^{\prime} 11^{\prime \prime W}, 5$ ninfas, F.F. Salles, J.D. Batista, H.R.S. Cabette.

COMENTÁRIOS: Exatamente como $W$. fasciatus, $Z$. principalis também se apresenta distribuída nas regiões Norte e Sudeste do país (Lugo-Ortiz \& McCafferty 1998, Lugo-Ortiz et al. 2002, Salles et al. 2003b). E, da mesma forma, mesmo o registro do gênero e da espécie sendo inéditos para toda a Região Centro-Oeste, sua presença já era esperada.

\section{Discussão}

Como pôde ser demonstrada, a fauna de Baetidae do Estado do Mato Grosso apresenta uma diversidade, até o momento ao menos com relação aos gêneros, bastante elevada. Mesmo possuindo um número reduzido de trabalhos sobre esse assunto (e.g. McCafferty \& Lugo-Ortiz 1995, Nolte et al. 1997, Lugo-Ortiz \& McCafferty 1998, Salles \& Lugo-Ortiz 2002a, Salles \& Batista, 2004), o número de gêneros agora reportados para o estado é significativamente elevado. Iguaira Salles \& Lugo-Ortiz, 2003, Moribaetis Waltz \& McCafferty, 1985, Rivudiva Lugo-Ortiz \& McCafferty, 1998, Tomedontus Lugo-Ortiz \& McCafferty, 1995 e Tupiara Salles, Lugo-Ortiz, Da-Silva \& Francischetti, 2003, são os únicos dos atuais vinte gêneros de Baetidae reportados para o Brasil não encontrados no estado.

Como os trabalhos referentes à fauna de Baetidae são absolutamente pontuais, restritos ao centro-sul e sudeste do estado, esforços amostrais em outras áreas do Mato Grosso são ainda extremamente necessários e devem aumentar consideravelmente o número de espécies e talvez até de gêneros nele encontrados. Não só com relação a novos registros, mas o número de espécies por descrever também deve ser alto, como aponta o presente trabalho (além de $C$. auwe sp. nov., espécies desconhecidas de Apobaetis e Cryptonympha também foram encontradas, apesar de não terem sido descritas).

\section{Agradecimentos}

A Marcelo da Silva Baptista, Universidade Federal de Viçosa, por valiosas sugestões ao manuscrito, ao biólogo Lourivaldo Amancio de Castro e a equipe do Laboratório de Entomologia da Universidade do Estado de Mato Grosso, Campus de Nova Xavantina, pelo auxílio na realização das coletas. Ao CNPq, por prover fundos ao primeiro autor como estudante do Programa de Pós-graduação em Entomologia na Universidade Federal de Viçosa e apoio financeiro às viagens de campo, CNPq proc.550490/01-9.

\section{Referências bibliográficas}

DA-SILVA, E.R. 1991. Descrição da ninfa de Callibaetis guttatus Navás, 1915, com notas sobre a imago (Ephemeroptera: Baetidae). An. Soc. Entomol. Brasil 20: 345-352.

DAY, W.C. 1955. New genera of mayflies from California (Ephemeroptera). Pan-Pacific Entomol. 31:121-137.

DEMOULIN, G. 1955. Une mission biologique belge au Brésil. Éphéméroptères. Bull. Inst. R. Sci. Nat. Belg. 31(20): 132.

DOMINIQUE, Y., THOMAS, A.G.B., ORTH, K. \& DAUTA, C. 2000. Les Ephémères de la Guyane Francaise. 2. Camelobaetidius billi et C. janae n. spp. [Ephemeroptera, Baetidae]. Ephemera 2(1)2000: 39-48.

EATON, A.E. 1881. An announcement of new genera of the Ephemeridae. Entomologist's Mon. Mag. 17:191-197.

FRANCISCHETTI, C.N., SALLES, F.F., DA-SILVA, E.R. \& LUGO-ORTIZ, C.R. 2003. First report of Americabaetis Kluge (Ephemeroptera: Baetidae) from Rio de Janeiro, Brazil. Entomotropica 18(1): 69-71.

LUGO-ORTIZ, C.R. \& McCAFFERTY, W.P. 1995. Three distinctive new genera of Baetidae (Insecta: Ephemeroptera) from South America. Annls. Limnol. 31(4):233-243.

LUGO-ORTIZ, C.R. \& McCAFFERTY, W.P. 1996a. Aturbina georgei gen et $\mathrm{sp}-\mathrm{n}$. A small minnow mayfly (Ephemeroptera, Baetidae) without turbinate eyes. Aquat. Insects 18:175-183.

LUGO-ORTIZ, C.R. \& McCAFFERTY, W.P. 1996b. The genus Paracloeodes (Insecta, Ephemeroptera, Baetidae), and its presence in South America. Annls. Limnol. 32(3):161169.

LUGO-ORTIZ, C.R. \& McCAFFERTY, W.P. 1996c. Taxonomy of the Neotropical genus Americabaetis, new status (Insecta: Ephemeroptera Baetidae). Stud. Neotrop. Fauna Environ. 31:156-169.

LUGO-ORTIZ, C.R. \& McCAFFERTY, W.P. 1997. First report and new species of the genus Apobaetis (Ephemeroptera: Baetidae) from South America. Aquat. Insects 4:243-246. 
LUGO-ORTIZ, C.R. \& McCAFFERTY, W.P. 1998. Five new genera of Baetidae (Insecta: Ephemeroptera) from South America. Annls. Limnol. 34(1):57-73.

LUGO-ORTIZ, C.R., SALLES, F.F. \& FURIERI, K.S. 2002. First records of small minnow mayflies (Ephemeroptera: Baetidae) from the state of Espírito Santo, southeastern Brazil. Lundiana 3: 79-80.

MAYO, V.K. 1972. New species of the genus Baetodes (Ephemeroptera: Baetidae). Pan-Pac. Entomol. 48(4):226241.

McCAFFERTY, W.P. \& LUGO-ORTIZ, C.R. 1995. Cloeodes hydation, n. sp. (Ephemeroptera: Baetidae): an extraordinary drought tolerant mayfly from Brazil. Entomol. News 106:29-35.

MOL, A.W.M. 1986. Harpagobaetis gulosus gen. nov., spec. nov., a new mayfly from Suriname (Ephemeroptera: Baetidae). Zool. Meded. 4:63-70.

NEEDHAM, J.G. \& MURPHY, H.E. 1924. Neotropical mayflies. Bull. Lloyd Library Botany, Pharmacy Materia Medica No 24, Entomol. Ser. 4:1-79.

NOLTE, U., OLIVEIRA, M.J. \& STURS, E. 1997. Seasonal, discharge-driven patterns of mayfly assemblages in an intermittent Neotropical stream. Freshwater Biology 37:333-343

ORTH, K., THOMAS, A.G.B., DAUTA, C., HOREAU, V., BROSSE, S. \& ADEMMER, C. 2000. Les Ephémères de la Guyane Française. 1. Premier inventaire générique, à but de biosurveillance [Ephemeroptera]. Ephemera 2: 2538.

SALLES, F.F. \& BATISTA, J.D. 2004. The presence of Varipes Lugo-Ortiz \& McCafferty (Ephemeroptera: Baetidae) in Brazil, with the description of a new species. Zootaxa 456: $1-6$.

SALLES, F.F. \& FRANCISCHETTI, C.N. 2004. Cryptonympha dasilvai sp. nov. (Ephemeroptera: Baetidae) do Brasil. Neotropical Entomology 33(2): 213216.

SALLES, F.F. \& LUGO-ORTIZ, C.R. 2002a. A distinctive new species of Apobaetis (Ephemeroptera: Baetidae) from Mato Grosso and Minas Gerais, Brazil. Zootaxa 35: 1-6.

SALLES, F.F. \& LUGO-ORTIZ, C.R. 2002b. Primeiro registro do gênero Harpagobaetis Mol (Ephemeroptera: Baetidae) para o Brasil. Lundiana 3: 155.

SALLES, F.F. \& LUGO-ORTIZ, C.R. 2003. Nova espécie de Cloeodes Traver (Ephemeroptera: Baetidae) do Estado do Rio de Janeiro. Neotropical Entomology 32: 449-453.

SALLES, F.F, DA-SILVA, E.R. \& LUGO-ORTIZ, C.R. 2003a. Descrição da ninfa e redescrição dos adultos de Callibaetis radiatus Navás (Insecta: Ephemeroptera: Baetidae). Lundiana 4(1): 13-18.
SALLES, F.F., FRANCISCHETTI, C.N., ROQUE, F.O., PEPINELLI, M. \& TRIVINHO-STRIXINO, S. 2003b. Levantamento preliminar dos gêneros e espécies de Baetidae (Insecta: Ephemeroptera) do Estado de São Paulo, com ênfase em coletas realizadas em córregos florestados de baixa ordem. Biota Neotropica 3(2): http:/ /www.biotaneotropica.org.br/v3n2/pt/abstract?shortcommunication+BN01103022003

TRAVER, J.R. 1938. Mayflies of Puerto Rico. J. Agric. Univ. Puerto Rico. 22:5-42.

TRAVER, J.R. 1944. Notes on Brazilian mayflies. Bol. Mus. Nac., N.S., Zool. 22:2-53.

WALTZ, R.D. \& McCAFFERTY, W.P. 1985. Moribaetis: A new genus of Neotropical Baetidae (Ephemeroptera). Proc. Entomol. Soc. Wash. 87:239-251.

Título: Baetidae (Insecta: Ephemeroptera) de Nova Xavantina, Mato Grosso, Brasil: novos registros e descrição de uma nova espécie de Cloeodes Traver

Autores: Frederico Falcão Salles; Joana Darc Batista; Helena Ramos Soares Cabette

Biota Neotropica, Vol. 4 ( número 2): 2004

http://www.biotaneotropica.org.br/v3n 1/pt/ abstract?article+BN00303012003

recebido em: 19/4/2004 - revisado em: 18/6/2004

publicado em: 01/07/2004

ISSN 1676-0603 